\title{
Formulation of Two-Stage Stochastic Programming with Fixed Recourse
}

\author{
Hashnayne Ahmed \\ Department of Mathematics, University of Barishal, Barishal-8200, Bangladesh \\ hashnayneahmed17@gmail.com
}

Abstract: Stochastic Programming is an asset for the next world researchers due to its uncertainty calculations, which has been skipped in deterministic world experiments as it includes complicated calculations. Two-stage stochastic programming concerns two time period decisions based on some random parameters obtained from past experience or some sort of survey. The objective function for formulating two-stage stochastic programming with fixed recourse includes two parts: first-stage forecast and second-stage fixed decisions based on the experiment results. The constraints are similar to the normal optimization techniques rather some adjustments of requirements and technology assets. The fixed recourse decisions are sort of decisions from the deterministic world. Formulation techniques of two-stage stochastic programming with fixed recourse may be used for further complications arises in stochastic programming like complete recourse problems, multi-stage problems, etc. And that's why Two-stage stochastic programming with fixed recourse is called the primary model for stochastic programming.

Keywords: Stochastic Optimization; Stochastic Programming; Two-Stage Formulation; News Vendor Problem; Fixed Recourse

\section{Introduction}

Stochastic programming is studying optimization techniques under some sort of risk. The risk may also define as uncertainty since the risk occurs due to some uncertain parameters. Optimization techniques help to make the best possible decision for us and stochastic programming also does the same phenomena with uncertainty. It deals with the cases where uncertain parameters arise. It is required an expectation value to calculate the objective function in its exact value, which may also call the probability value. The probability distribution along with the uncertain parameters may calculate the exact randomness for a phenomenon. Dealing with these phenomena with uncertainty, arises different types of programs and this different type of programs has a different methodology for further experiment.

Two-stage stochastic programming is one mostly used kind of stochastic programming problems where the decision-maker has to make decisions in two stages (two different times) for a fixed phenomenon with uncertainty. The first stage decision plays a vital role here since this decision has to anticipate based on some random parameters obtained from past experience or some sort of survey. And the second stage decision is based on the experiment result or some anticipation. Two-stage stochastic programming with fixed recourse or simple recourse simply discuss the phenomena with simple second-stage decisions which could be fixed under some circumstances. In making the first stage decisions, second stage forecast with some sort of possibility (mathematical expectation) has to be taken into account.

Two-stage stochastic programming problem is the primary model for uncertainty computation. It could be used for representing multiple stages of decisions with block separable recourse, which is the foundation of multi-stage stochastic programming.

There are many applications of the stochastic process since uncertainty is everywhere. All the deterministic experiments occurred in this world are calculated based on some certain parameters, actually some certain state of this uncertain world. It will be more beneficial for us in the long run if we take uncertain issues everywhere. It will work like some insurance policy for the applications which may cost a little but in the long run, it will be some kind of blessings. Stochastic 
programming is vastly used in different optimization technique applications areas like finance, technology, ecology, maintenance, arc routing, etc. (Gassmann, 2005; Ahmed, 2019).

\section{Theoretical Review}

\subsection{Objective}

Linear Programs normally targets a minimum cost solution with some requirements or demands or a maximum profit solution under given requirements. Stochastic linear programs are also linear programs in which some required data may be uncertain (means some problem data is random variable). Recourse Program means the program needs some new decisions or recourse actions after the uncertainty is no longer concealed.

\subsection{Decision and Stages}

A set of decisions could be taken for stochastic linear programs. We may divide them into two sections: (Birge, 2011; Kall, 1976)

Some decisions have to be taken before the experiment. In this case, all the decisions are called first stage decisions and the decision-making period is called the first stage.

Some decisions have to be taken after the experiment. In this case, all the decisions are called second stage decisions and the decision-making period is called the second stage.

Here some particular values of random variables will be unveiled after the experiment. We may express those random variables using $\xi(\omega)$, where $\omega$ is the outcome of an uncertain random experiment. If we denote the first stage decisions using $x$ and the second stage decisions using $y$ or $y(\omega)$ or $y(\omega, x)$ since the values of $y$ depends on the outcome of the experiment, then we may summarize the sequence of decisions as

For example,

$$
x \stackrel{\text { yields }}{\longrightarrow} \xi(\omega) \stackrel{\text { yields }}{\longrightarrow} y(\omega, x)
$$

If a farmer wants to raise crops, he has to consider the forecast weather conditions. In that case, the first stage resembles planting and the second stage decisions comprised of sales.

A product deliveryman travels in a sequential way every time, which is the first stage decision but if the product lacks after some clients then he has to second stage decisions.

A student makes his exam preparation with first stage choice but after getting the question papers he has to decide about answering questions, which is the second stage decisions.

\subsection{Formulation}

As briefed earlier, we have to make some decisions before the experiment (without full information) on some random events. These are the first stage decisions denoted by a vector $x$. Afterward, we get the results of the experiment considering some random variables $\xi(\omega)$. Then we take the second stage decisions $y(\omega)$. Using Mathematical Programming concepts, this defines the alleged two-stage stochastic program with the recourse of the following form (accredited by Dantzig and Beale, 1955):

$$
\begin{gathered}
\text { Min } z=c^{T} x+E_{\xi} Q(x, \xi) \\
\text { Subject to, } A x=b \\
x \geq 0 .
\end{gathered}
$$

Where the first term of the objective function represents the first stage decisions and the second term represents the second stage decisions.

Here,

$$
Q(x, \xi)=\min \left\{q^{T} y \mid W y=h-T x, y \geq 0\right\}
$$

$c$ are the first stage constraints.

$q$ are the second stage constraints. 
$E_{\xi}$, are the Mathematical Expectations with respect to random vector $\xi$.

$W$, are the fixed recourse.

$h(\omega)$, are the requirements.

$T(\omega)$, are the Technology Matrix.

For a given outcome $\omega, Q(x, \xi(\omega))$ is called the second stage value function defined as the formula (2).

Let us define the Expected second stage value function or recourse function

$$
\mathrm{Z}(x)=E_{\xi} Q(x, \xi(\omega))
$$

Using this we get the so-called deterministic equivalent program (DEP) as follows:

$$
\begin{gathered}
\text { Min } z=c^{T} x+\mathrm{Z}(x) \\
\text { Subject to, } A x=b \\
x \geq 0 .
\end{gathered}
$$

Here, if the expected second stage value function is given then the stochastic program becomes an ordinary non-linear program.

In the above representations, $x$ are taken in the presence of uncertainty about future awareness of $\xi$. Then the actual value of $\xi$ becomes known after certain experiments and recourse decisions $y$ are taken. First stage decisions are preferred by considering future effects. These future effects are measured by the value function or recourse function $\mathrm{Z}(x)$, which is the required value for taking decision $x$.

Another case could occur. If the Technology Matrix $T$ is non-stochastic, then we may get the following formulation: (Birge, 2011)

$$
\begin{aligned}
& \text { Min } z=c^{T} x+\Psi(\chi) \\
& \text { Subject to, } A x=b \\
& \text { Tx } x=0 \\
& \text { And } x \geq 0
\end{aligned}
$$

where,

and

$$
\Psi(\chi)=E_{\xi} \psi(\chi, \xi(\omega))
$$

$$
\psi(\chi, \xi(\omega))=\min \left\{q^{T} y \mid W y=h-\chi, y \geq 0\right\}
$$

Thus, finally, we may say that solving stochastic programming clearly lies in the computational burden of recourse function $\mathrm{Z}(x)$ for all $x$, otherwise $\Psi(\chi)$ for all $\chi$.

\subsection{General Extensive Form}

When we have a finite number of scenarios, or if we approximate the problem with a finite number of scenarios, we can write an equivalent extensive form linear program: (Kall, 1976)

Subject to

$$
\operatorname{Min} c^{\mathrm{T}} \mathrm{x}+\mathrm{E}_{1} \mathrm{q}_{1}^{\mathrm{T}} \mathrm{y}_{1}+\mathrm{E}_{2} \mathrm{q}_{2}^{\mathrm{T}} \mathrm{y}_{2}+\cdots+\mathrm{E}_{\mathrm{s}} \mathrm{q}_{\mathrm{s}}^{\mathrm{T}} \mathrm{y}_{\mathrm{s}}
$$

where $x \in X, y \in Y$.

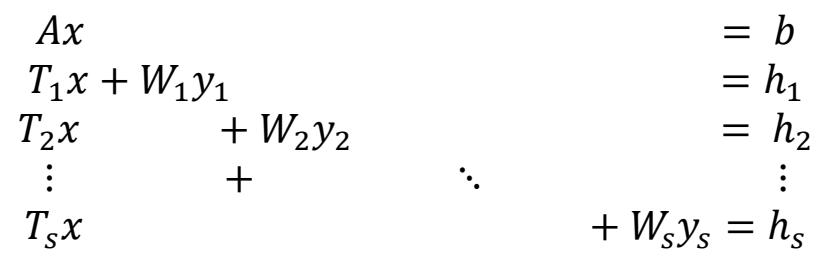

\subsection{Discrete Random Variable}


We will now classify the decision variables with the configuration of randomness. Let us define the following two sets: (Birge, 2011)

$K_{1}=\{x \mid A x=b, x \geq 0\}$ i

$$
K_{2}(\xi)=\{x \mid y \geq 0 \text { where } W(\omega) y=h(\omega)-T(\omega) x\}
$$

Here the set $K_{1}$ is the set determined by fixed constraints, does not depend on any random decision. And the set defined by $K_{2}$ is randomness-based decisions for any given $\xi$.

Discrete random variables are widely used in applications, either directly or through the sampling of a continuous distribution.

\section{Discussion}

a. A two-stage stochastic optimization problem has fixed recourse or simple recourse if for every scenario, the coefficients of the second stage variables are deterministic. (Birge, 2011; Kall, 1976)

b. A two-stage stochastic optimization problem has complete recourse if, for every scenario, there always exists a feasible second solution: (Birge, 2011; Kall, 1976)

$$
Q_{s}(x)<\infty, \quad \forall x \in R \text { and } s=1,2, \ldots, S
$$

c. A two-stage stochastic optimization problem has relatively complete recourse if for every scenario, and for every feasible first stage solution, there always exists a feasible second solution: (Birge, 2011; Kall, 1976)

$$
Q_{s}(x)<\infty, \quad \forall x \in X \text { and } s=1,2, \ldots, S
$$

\section{Conclusion}

This paper is about the formulation of the most basic stochastic programming problem of the two-stage stochastic linear program with fixed recourse kind. Here the second-stage decisions are from the deterministic world and that implies all the importance to the first-stage forecast. Formulation of two-stage stochastic programming with fixed recourse calculates the first-stage forecast and some kind of second-stage anticipation. All the decision variables could be classified into two sets: fixed constraint variables and discrete random variables; for the further experiment on stochasticity. This formulation technique is the basic foundation of stochastic programming.

\section{Acknowledgments}

The authors would like to thank respectable Professor John Birge for such a nice and insightful book on Stochastic Programming.

\section{References}

Birge, John R., and Francois Louveaux. Introduction to stochastic programming. Springer Science \& Business Media, 2011.

Kall, Peter, and Janos Mayer. Stochastic linear programming. Vol. 7. Berlin: Springer-Verlag, 1976.

Dantzig, George B. "Linear programming under uncertainty." Stochastic programming. Springer, New York, NY, 2010. 1-11.

Ahmed, Hashnayne. "Graph Routing Problem Using Euler's Theorem and Its Applications." (2019).

Beale, E. M. L., George B. Dantzig, and R. D. Watson. "The first-order approach to a class of multitime-period stochastic programming problems." Stochastic Programming 84 Part I. Springer, Berlin, Heidelberg, 1986. 103-117.

Gassmann, Horand I., et al. "Introduction to stochastic programming applications." Applications of stochastic programming. Society for Industrial and Applied Mathematics, 2005. 179-184. 